\title{
Charts for Exponentially Weighted Moving Average Classical and Robust: A Comparative Study
}

\author{
Aida Hadi Saleh ${ }^{\text {a,* }}$ \\ ${ }^{a}$ Statistics Department, Administration \& Economics College, AL-Mustansiriyah University, Iraq \\ E-mail: "aida.stat@yahoo.com
}

\begin{abstract}
This research discusses the comparison between two control charts classical \& robust for both types (EWMA-SMQ) and (EWMA-SM) for the exponentially weighted moving average, which is showed that the robust (EWMA-SMQ) chart for the process is a superior alternative to the (EWMA-SM) chart when the outliers are present in the data. Generally, the (EWMA-SMQ) chart enables easier detection of outliers in the subgroups and is also more sensitive to other forms of out-of-control situations when outliers are present. Hence, the (EWMA-SMQ) becomes a preferred alternative to be taken and applied in quality control. The data used in this research represent the weights of the Al-Sabah Iraqi newspaper published by the Iraqi media networks. Twenty-five samples were taken and each sample consisted of five observations. These samples were taken at different production times, as the average weight of the newspaper was approximately (150) g. Through the application of the classical control chart (EWMA), exit four points for the upper and lower control limits, and the application of (EWMA-SM), (4) points are out of the upper and lower limits of the control; the control limits have been extended from the top and bottom sides of the (EWMA-SM) chart, making it less sensitive to the diagnosis of the shift in the mean process. The (EWMA-SMQ) chart detected extra points out of control. There were (7) points out of the upper and lower limits for the control, tight control limits of the (EWMA-SMQ) chart from the top and bottom sides. Due to this narrowness, three additional points were detected. Therefore (EWMA-SMQ) chart is more robust than the (EWMA-SM) control chart. This research aims to make a comparative study between the classical and robust for both types (EWMA-SM) \& (EWMA-SMQ) for the exponentially weighted moving average control chart. The most important result of the research that the (EWMA-SMQ) chart is more suitable than the (EWMA-SM) \& classical (EWMA) when outliers are present in the data. This gives importance to it to control the quality of the product. By this robust chart, the outliers must be detected, investigated and the special cause removed if possible. The presence of outliers will reduce the sensitivity of a control chart. The (EWMA-SMQ) control chart is more sensitive to out-of-control conditions when outliers are present in data. The limits computed from the estimate of the interquartile ranges for the (EWMA-SMQ) chart are less influenced by outliers than the (EWMA-SM) chart where the limits are computed based on the sample ranges. Thus the (EWMASMQ) chart is more robust than the (EWMA-SM) chart.
\end{abstract}

Keywords— EWMA-SM; EWMA-SMQ; average robust; robust chart; classical chart.

Manuscript received 7 Jul. 2020; revised 24 Dec. 2020; accepted 5 Jan. 2020. Date of publication 28 Feb. 2021. IJASEIT is licensed under a Creative Commons Attribution-Share Alike 4.0 International License.

\section{INTRODUCTION}

The statistical tools are used to control the quality of the output and to monitor its control charts. The concept of quality is occupied a wide scope in productive, administrative, and practical applications, and the quality control charts were widely used in industries. The purpose of their use is to monitor processes and detect the out-of-control patterns. Power control charts lie in their ability to diagnose the shift in processes and to distinguish abnormal situations. Some previous studies designed the first control chart, and several attempts were appeared to develop what began Shewhart [1], [2]. The focus was on what goes wrong on
Shewhart's chart that it is less sensitive in detecting of small continuous and medium changes the average process changed, and try to reduce the limits of control to less than three standard deviations from the use of scientific methods. The scientist has moved to moving and weighted average methods to reduce control limits, helping to reduce differences between values, which reduces their deviations. Along with extensive use of the chart, other types of charts appeared like exponentially weighted moving average charts developed [3], the basic goal of this chart is to detect small shifts in the control process's goal. Various robust control charts appeared, such as a robust exponentially weighted 
moving average, and the robustness is the right key for proper design and application, and implementation. Since Shewhart chart proved to be inefficient in detecting small changes in the average process, they were limited to detecting large deviations only. The alternative solution that treats this situation is using control charts exponentially weighted moving average classical and robust within the same objective. It is subject to detect small deviations in the process of productivity that does not appear in the Shewhart chart. It deals with current and past observations of the production process. The main objective of this research is to make a comparative study between the traditional and robust control charts for the exponentially weighted moving average. Actual data were used to represent the weights of Al-Sabah Iraqi newspaper for twenty-five samples of this research, every sample consisted of five observations, these samples were taken at different times.

Previous research also provided a run-length distribution of exponentially weighted moving average charts and presented average operating length (ARL) and standard deviation of operation lengths [3]. These were subject to assume a normal distribution of observations and then expanded the study for many abnormal distribution cases. Another study [4] completed scales in the rate of operating length and graphics in the selection of optimum values for control charts parameters of (EWMA) in designing the (EWMA) control chart. Abbas [5] presented several (EWMA) control chart schemes based on individual measurement, the sample mean, sample range, and moving range statistics. In 1990, the (EWMA) control chart was studied, and it was concluded that the chart was useful in detecting cyclical changes in the average process [6]. It showed that a fast initial response (FIR) feature is useful for the (EWMA) chart, especially for small values of smoothing constants. Raza [7] proposed a new (EWMA) control chart based on (SC) statistic that the area below the straight line is a control area that makes the control method simpler than other methods and can detect the source and direction of the change that occurs during the sampling period. Each of them found that this chart was more efficient than the Shewhart chart in detecting random deviations that occur during the production processes.

Some previous studies concerned the design and optimization of the (EWMA) control chart for areas under control and outside under normal distribution assumption [8], [9]. Sharaf [10] used the (EWMA) chart for individuals indicating that this chart can be considered a new weighted average of all previous and current observations, and it is not sensitive to normal assumption and therefore is a perfect chart for individual observations. The robustness of the (EWMA) control chart for individual observations was observed where the (EWMA) chart is more robust to the small values of the smoothing parameter $(\lambda)[11]$. (EWMA) The control chart was modified, and the modification effectively detected the sudden change in the average process [12]. The benefit of using the modified (EWMA) chart lies in a good performance when the observations follow the normal distribution, whether independent or correlated.

The (EWMA) charts were developed [13] under different robust scale estimators (i.e., Gini's mean difference $(G), S_{n}$, $\mathrm{Q}_{\mathrm{n}}, \mathrm{MAD}$. The performance of these charts were compared by calculating expected out of control points and expected widths under non-symmetric distribution (i.e., Gamma \& exponential) [14] [15]. This simulation study showed that among six robust estimators, the chart based on estimator $Q_{n}$ relatively performed well for non-normal processes in terms of its shorter expected width and more expected out of control points, showing its sensitivity to detect the out-of-control signal.

\section{Materials AND Methods}

\section{A. Classical Exponentially Weighted Moving Average Chart}

Control charts (EWMA) for the process-mean, provide us more sensitivity for the small shifts. It is more than the Shewhart chart for the arithmetic mean. It is a good alternative to the Shewhart chart when our attention focuses on detecting small shifts. It has flexibility in the calculation by choosing a weight factor and use this factor to balance primary and recent data [16].

The construction of (EWMA) is concerned with monitoring the mean of any production process expressed by $\left(\mathrm{Z}_{\mathrm{i}}\right)$ using weighting weight according to the following formula:

$$
(E W M A)_{i}=Z_{i}=\lambda x_{i}+(1-\lambda) Z_{i-1}
$$

Where $(\lambda)$ represents a smoothing constant, whose value is determined by the period $(0<\lambda \leq 1)$, small values of $(\lambda)$ is useful in diagnosing small shifts in the process, and large values are useful in diagnosing large shifts in the process. $Z_{0}=\mu_{0}$, so that the starting point $Z_{0}$ can be taken as the average initial value or $Z_{0}=\overline{\bar{x}}$ if there are subgroups.

$Z_{i}$ : The mean of historical data (Target).

$x_{i}$ : The observations at the time.

$n$ : The number of the observations to be monitored.

$\lambda$ : Smoothing Constant.

A value of $\lambda=1$ implies that only the most recent measurement influences the (EWMA). Thus, a considerable value of $\lambda$ (closer to 1 ) gives more weight to recent data and less weight to older data, a small value of $\lambda$ (closer to 0 ) gives more weight to older data. The value of $\lambda$ is usually set between ( 0.2 and 0.3$)$, although this choice is somewhat arbitrary [16] give tables that help the user select $\lambda$.

Sequential values for $Z_{i}$ where $\mathrm{i}=1,2, \ldots, \mathrm{n}$ named the exponentially weighted moving average. If the observations are randomly independent in contrast $\sigma^{2}, Z_{i}$ would be:

$$
\sigma_{Z_{i}}^{2}=\sigma^{2}\left(\frac{\lambda}{2-\lambda}\right)\left[1-(1-\lambda)^{2 i}\right]
$$

and therefore, the limits of control chart (EWMA) are:

$$
U C L=\mu_{0} \sqrt{\frac{\lambda}{2-\lambda}\left[1-(1-\lambda)^{2 i}\right]}
$$

Either if we have subgroups equal to (m), and each subgroup consist of number of observation equal to (n), we compute the arithmetic mean for the subgroups $\left(\bar{x}_{i}\right)$ (The arithmetic mean for the subgroup i), the formula of $Z_{i}$ is as follows :

$$
(E W M A)_{i}=Z_{i}=\lambda \bar{x}_{i}+(1-\lambda) Z_{i-1}
$$


The starting point will be $(E W M A)_{0}=\mu_{0}=\overline{\bar{x}}$, where that $\overline{\bar{x}}=\frac{\sum_{i=1}^{m} \bar{x}_{i}}{m}$. The control limits are in the case of averages for subgroups $\left(\bar{x}_{i}\right)$ is

$$
U C L=\overline{\bar{x}}+3 \sigma_{1} \sqrt{\frac{\lambda}{n(2-\lambda)}\left[1-(1-\lambda)^{2 i}\right]}
$$

$L C L=\overline{\bar{x}}-3 \sigma \sqrt{\frac{\lambda}{n(2-\lambda)}\left[1-(1-\lambda)^{2 i}\right]}$

The standard

deviation is obtained in several ways:

- If $\sigma$ is known, it will be compensated.

- It will be estimated by using the range, $\hat{\sigma}=\frac{R}{d_{2}}$, where $\mathrm{R}$ represents the difference between the largest value and the lowest value, $\mathrm{d}_{2}$ is fixed control. When we have several samples, and each has a range, the mean range of the samples is $\bar{R}$, and the standard deviation is estimated according to the formula $\hat{\sigma}=\frac{\bar{R}}{d_{2}}$.

- Are estimated using the standard deviation of the samples according to $\hat{\sigma}=\frac{\bar{S}}{C_{4}}$ where $\bar{S}$ represent the mean and standard deviation of the samples, $\mathrm{C}_{4}$ is the fixed control.

\section{B. The Robust Exponentially Weighted Moving Average chart}

We mentioned that the (EWMA) chart is a good alternative to the Shewhart chart in diagnosing small shifts in the process. The outliers consist of exceptional single values that occur for special reasons, these outliers must be diagnosed and verified and the cause removed if possible. The presence of outliers reduces the sensitivity of a control chart because the control limits extend. Thus, the detection of outliers become more difficult. Moreover, the extended limits make them more difficult for other types of signals out of control to be detected. Therefore, the robust (EWMA) control chart suggested for the mean process to diagnose easier for the outliers and increase sensitivity to other causes out of control when there are outliers. These outliers must be detected, investigated and the special cause removed if possible. Outliers' presence will reduce the sensitivity of a control chart because the control limits are stretched so that the outliers' detection becomes difficult.

Furthermore, these stretched limits also make it more difficult for other types of out-of-control signals to be detected. Suggestion a robust (EWMA) chart (EWMA-SMQ) chart for the process mean as a superior alternative to the standard EWMA (EWMA-SM) for the process mean. The (EWMA-SMQ) chart is constructed based on the limits that are set using an estimate of the process standard deviation using the average of the subgroup's interquartile ranges rather than the average of the subgroups range in the case of the (EWMA-SM).

\section{The Robust (EWMA-SM) chart}

The (EWMA-SM) chart is based on the following statistic

$$
(E W M A-S M)_{i}=\alpha \bar{x}_{i}+(1-\alpha)
$$

Where $\bar{x}_{i}$ is the mean of sample $\mathrm{i}$ and $(\alpha)$ is a weighting constant. In the estimation of the limits based on the process data, (m) subgroups of size (n) are taken, the average of (m) sample means is $\overline{\bar{x}}, \overline{\bar{x}}$ is used as the starting point, (EWMA$S M)_{0}=\overline{\bar{x}}$. The average of the $(\mathrm{m})$ sample ranges is computed to be $(\bar{R})$.

The upper and lower control limits of the (EWMA-SM) chart are:

$$
L C L=\overline{\bar{x}}-F_{1} \bar{R}
$$

Where

$$
F_{1}=\frac{3}{d_{2} \sqrt{n}} \sqrt{\frac{\alpha}{2-\alpha}}
$$

Where $d_{2}$ is a standard constant whose value depends on the sample size $(\mathrm{n})$.

\section{The Robust (EWMA-SMQ) Chart}

The (EWMA-SMQ) control chart depends on the same statistics as the previous one mentioned in the equation (6). We rewrite it in an accordance with the (EWMA0SMQ) control chart as follows:

$$
(E W M A-S M Q)_{i}=\alpha \bar{x}_{i}+(1-\alpha)
$$

Where $\bar{x}_{i}$ represents the mean of sample $\mathrm{i},(\mathrm{m})$ subgroups of size (n) each must be taken to compute the estimate of the process mean. The average of $(\mathrm{m})$ sample mean will be used as the starting point in computing (EWMA-SMQ) ${ }_{i}$ chart, i.e. $(E W M A-S M Q)_{0}=\overline{\bar{x}}$.

In (EWMA-SMQ) chart, the interquartile range (IQR) is defined as $x_{b}-x_{a}$, where it is order statistics, $a=\left[\frac{n}{4}\right]$ and $\mathrm{b}=\mathrm{n}-\mathrm{a}+1$. The mathematical expectation of (IQR) can be de defined as

$$
E(I Q R)=d_{2}^{Q} \sigma_{x}
$$

Where $d_{2}^{Q}$ is a constant whose value depends on the sample size $(\mathrm{n})$, it is shown in table (1). The standard deviation $\left(\sigma_{x}\right)$ is estimated by:

$$
\hat{\sigma}_{x}=\frac{\overline{I Q R}}{d_{2}^{Q}}
$$

Where $(\overline{I Q R})$ is the average of subgroup interquartile ranges. Assuming that all the observed data are independent from one sample to another, then

$$
\begin{gathered}
\operatorname{Var}(E W M A-S M Q)_{i}=\operatorname{Var}(\bar{x}) \frac{\alpha}{2-\alpha} \\
\sigma_{(E W M A-S M Q)_{i}}=\frac{\sigma_{x}}{\sqrt{n}} \sqrt{\frac{\alpha}{2-\alpha}}
\end{gathered}
$$

The equation (11) uses the average interquartile range to estimate the standard deviation of (EWMA-SMQ). The limits of the (EWMA-SMQ) control chart is:

$$
\begin{gathered}
U C L=\overline{\bar{x}}+3 \frac{\overline{I Q R}}{d_{2}^{Q}} \sqrt{\frac{\alpha}{2-\alpha}} \\
C C L=(E W M A-S M Q)_{0}=\overline{\bar{x}} \\
L C L=\overline{\bar{x}}-3 \frac{\overline{I Q R}}{d_{2}^{Q}} \sqrt{\frac{\alpha}{2-\alpha}}
\end{gathered}
$$

Accordingly, the calculated control limits based on equation (16) will be as follows:

$$
L C L=\overline{\bar{x}}-G_{1} \overline{I Q R}
$$

G1 for different samples sizes, (n) and smoothing constants. 


\section{RESULTS AND DISCUSSION}

The data used in this research represent the weights of AlSabah Iraqi newspaper published by the Iraqi media network, twenty-five samples were taken, and each sample consisted of five observations, these samples were taken at different times of production, as the daily weighted of the paper was approximately $(150) \mathrm{g}$. Table 1 represents the weight of the Iraqi newspaper Al-Sabah measured by grams.

TABLE I

THE WeIGHTS OF AL-SABAH NEWSPAPER

\begin{tabular}{|c|c|c|c|c|c|}
\hline \multirow{2}{*}{ Sample } & \multicolumn{5}{|c|}{ Processes } \\
\hline & $\mathrm{X}_{1}$ & $\mathrm{X}_{2}$ & $\mathrm{X}_{3}$ & $\mathrm{X}_{4}$ & $\mathrm{X}_{5}$ \\
\hline 1 & 150.3 & 148.8 & 148.6 & 148.1 & 148 \\
\hline 2 & 150.9 & 150.2 & 150.1 & 150.7 & 149.3 \\
\hline 3 & 146 & 145.7 & 146.8 & 148 & 146.4 \\
\hline 4 & 150.2 & 151.5 & 150.3 & 149.1 & 150.3 \\
\hline 5 & 149.1 & 148.4 & 148.7 & 150.7 & 150.8 \\
\hline 6 & 152.2 & 150 & 151.8 & 149.8 & 148.6 \\
\hline 7 & 147.1 & 148.4 & 149.3 & 149.5 & 147.7 \\
\hline 8 & 149.9 & 149.8 & 151.5 & 151.3 & 150.3 \\
\hline 9 & 149.7 & 149.3 & 147.9 & 148.4 & 148.7 \\
\hline 10 & 149.9 & 150.3 & 150.2 & 151.5 & 150.3 \\
\hline 11 & 145.9 & 146.3 & 145.7 & 145.5 & 146.3 \\
\hline 12 & 151.9 & 150.4 & 151.7 & 149.9 & 149.6 \\
\hline 13 & 146.9 & 148.9 & 147.8 & 148.2 & 147.8 \\
\hline 14 & 144.5 & 145.7 & 145.6 & 147.2 & 145.4 \\
\hline 15 & 149.6 & 148.6 & 150.6 & 150.4 & 148.7 \\
\hline 16 & 151.3 & 151.6 & 151 & 152.1 & 153.3 \\
\hline 17 & 149.2 & 149.9 & 149.4 & 150 & 148.2 \\
\hline 18 & 149.6 & 148.9 & 149.1 & 148.5 & 147.1 \\
\hline 19 & 145.4 & 145.8 & 146.3 & 147.6 & 148 \\
\hline 20 & 150 & 149.9 & 152.7 & 150 & 149.5 \\
\hline 21 & 147.6 & 146.2 & 147.9 & 147 & 147 \\
\hline 22 & 149 & 150.2 & 151.2 & 149 & 148.9 \\
\hline 23 & 150.7 & 150.8 & 151 & 152 & 151 \\
\hline 24 & 144.6 & 144.2 & 144.9 & 145.6 & 147.5 \\
\hline 25 & 150.4 & 151.3 & 152.8 & 150.7 & 150.9 \\
\hline
\end{tabular}

\section{A. Calculating the Classical (EWMA) control Chart}

From Table 2, we obtained the value of $\overline{\bar{x}}=149.0$, we take $\lambda=0.2$, using the equation (4), we obtained values of $(E W M A)_{i}$

For the first sample a value of $(E W M A)_{1}$ be

$$
\begin{aligned}
(E W M A)_{1}=Z_{1} & =(0.2)(148.76)+(0.8)(149.0) \\
& =148.95
\end{aligned}
$$

Similarly, we calculate the other samples, even the sample (25)

$$
\begin{gathered}
(E W M A)_{25}=Z_{25}=(0.2)(150.44)+(0.8)(148.48) \\
=148.87
\end{gathered}
$$

All values are shown in Table 2. The standard deviation is estimated according to $\hat{\sigma}=\frac{\bar{R}}{d_{2}}=\frac{2.164}{2.326}=0.93$ Where we obtained $\bar{R}=2.164$ from Table 2 , and so we obtained the value of $d_{2}=326$. Moreover, to calculate the classical (EWMA) chart's control limits, we use the equations mentioned in (5).

For the first sample, the control limits will be

$U C L_{1}=149.00+0.2494=149.25$

$C C L_{1}=149.00$

$L C L_{1}=149.00-0.2494=148.75$
Similarly, we calculate the control limits for the other sample and up to the twenty-five samples. The values of control limits are all shown in Table 3. Figure 1 shows the limits of control.

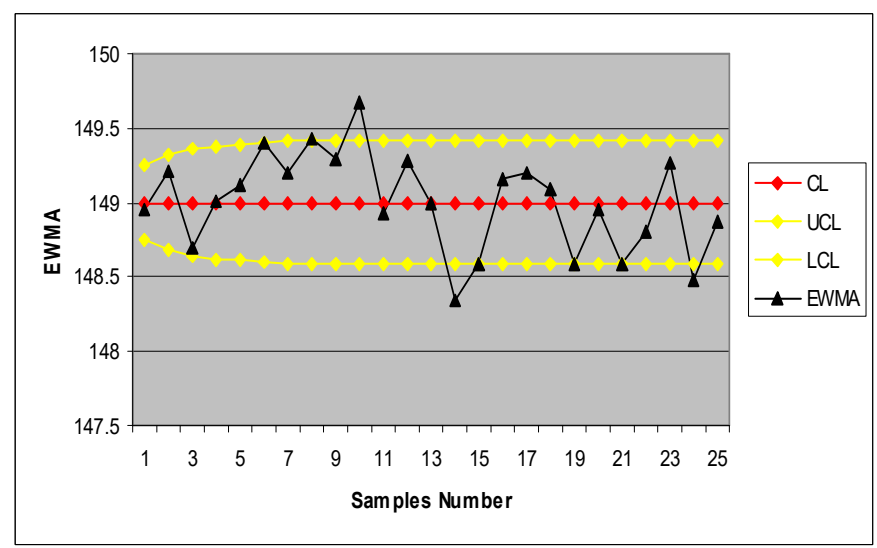

Fig.1 The control limits for the classical (EWMA) chart

Figure 1 shows that there are (4) points out of the upper and the lower control limits. The classical (EWMA) control chart detects out of control points at subgroups 8, 10, 14, and 24 .

\section{B. Calculating the Robust (EWMA) Control Chart}

From Table 2, we obtained the values of both

$$
\overline{\bar{X}}=149.00, \quad(E W M A)_{0}=\overline{\bar{X}}, \quad \bar{R}=1.64
$$

By using equation (6), (EWMA-SM) values can be obtained for twenty-five samples. These are the same values that we have obtained in the traditional (EWMA) chart application side. Using equation (7), we fined the robust (EWMA-SM) control limits. We obtained the value of $F_{1_{(\alpha=0.2, n=5)}}=0.192$, or can be obtained manually through the formula in equation (8) to find the control limits.

$$
U C L_{(E W M A-S M)}=149.00+(0.192)(2.164)=149.42
$$

$C C L_{(E W M A-S M)}=149.00$.

$L C L_{(E W M A-S M)}=149.00-(0.192)(2.164)=148.58$

Figure 2 shows the control limits for the robust (EWMA-SM) chart.



Fig. 2 The control limits for robust (EWMA-SM) chart

Figure 2 shows that there are four points out of the upper and lower control limits. The (EWMA-SM) control chart signals at subgroups $8,10,14$, and 24. Widening the (EWMASM) chart limits so that the chart is less sensitive in detecting shifts in the mean. 
C. Calculating the Robust (EWMA-SMQ) Control Chart

We obtained the values of $a=2$ and $b=4$; we can also find them manually where

$a=[n / 4]+1$

$a=[5 / 4]+1=2$

$b=n-a+1$

$b=5-2+1=4$

Interquartile Range $(\mathrm{IQR})=X_{(b)}-X_{(a)}$

$=X_{(4)}-X_{(2)}$

To find the interquartile ranges, the observations are arranged in each of twenty-five subgroups. By subtracting the $\mathrm{Q}_{2}$ from $\mathrm{Q}_{4}$ for all subgroups, we obtained the values of interquartile ranges. It is shown in the Table 2.

By using the equation (11), we obtained

$\hat{\sigma}_{X}=\frac{0.896}{0.9900}=0.9051$

By using equation (14) we obtained the value

$\hat{\sigma}_{(E W M A-S M Q)}=\frac{0.896}{0.9900(2.2361)}(0.3333)=0.1349$

To find the control limits, we used the equations (17), the value of $G_{1}=0.4517$. It can also be calculated manually according to the formula in equation (16). Thus, the control limits are as follows:

$U C L_{\left(E W M A_{-} S M Q\right)}=149.00+0.4517(0.896)=149.40$

$C C L_{(E W M A-S M Q)_{0}}=\overline{\bar{X}}=149.00$

$L C L_{\left(E W M A_{-} S M Q\right)}=149.00-0.4517(0.896)=148.60$

Figure (3) shows the control limits of the robust (EWMASMQ) chart.

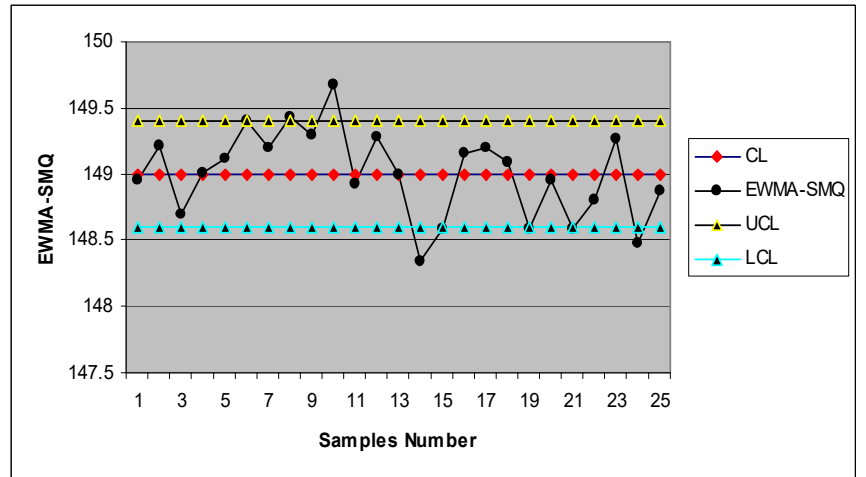

Fig 3. The control limits for robust (EWMA-SMQ) chart

Figure 3 shows that there are seven points out of the upper and lower control limit. The (EWMA-SMQ) control chart signals at subgroups $8,10,14,15,19,21$, and 24. The (EWMA-SMQ) control chart detects three additional out of control points (i.e., subgroups 15, 19, and 21) besides the four points at subgroups $8,10,14$, and 24 .
TABLE II

The Values Subgroups Sample Means, Sample Ranges, Sample INTERQUARTILE RANGES AND EWMA STATISTICS

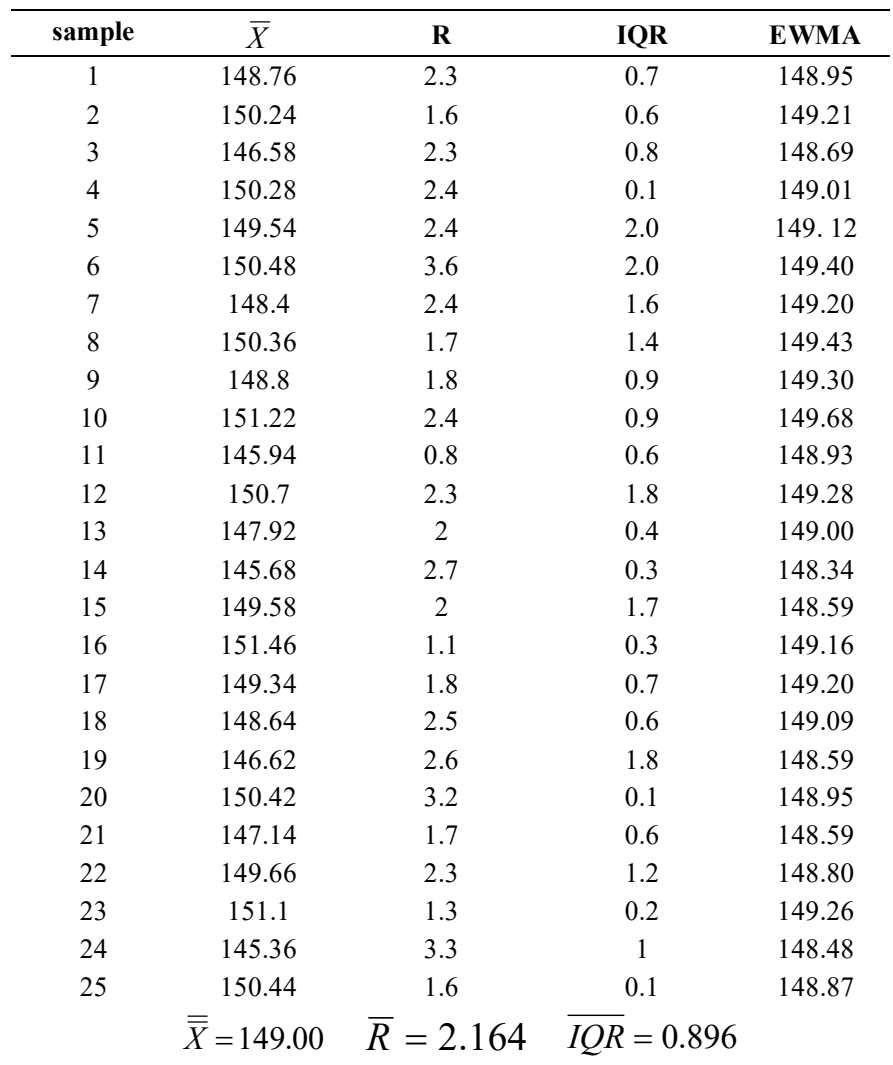

TABLE III

CONTROL LiMITS FOR THE ROBUST AND CLASSICAL CONTROL CHARTS

\begin{tabular}{ccccccc}
\hline Samples & \multicolumn{2}{c}{$E W M A_{C L}$} & \multicolumn{2}{c}{$E W M A_{S M}$} & \multicolumn{2}{c}{$E W M A_{S M Q}$} \\
\hline & $\mathbf{U C L}$ & $\mathbf{L C L}$ & $\mathbf{U C L}$ & $\mathbf{L C L}$ & $\mathbf{U C L}$ & $\mathbf{L C L}$ \\
1 & 149.25 & 148.75 & 149.42 & 148.58 & 149.40 & 148.60 \\
2 & 149.32 & 148.68 & 149.42 & 148.58 & 149.40 & 148.60 \\
3 & 149.36 & 148.64 & 149.42 & 148.58 & 149.40 & 148.60 \\
4 & 149.38 & 148.62 & 149.42 & 148.58 & 149.40 & 148.60 \\
5 & 149.39 & 148.61 & 149.42 & 148.58 & 149.40 & 148.60 \\
6 & 149.40 & 148.60 & 149.42 & 148.58 & 149.40 & 148.60 \\
7 & 149.41 & 148.59 & 149.42 & 148.58 & 149.40 & 148.60 \\
8 & 149.41 & 148.59 & 149.42 & 148.58 & 149.40 & 148.60 \\
9 & 149.41 & 148.59 & 149.42 & 148.58 & 149.40 & 148.60 \\
10 & 149.41 & 148.59 & 149.42 & 148.58 & 149.40 & 148.60 \\
11 & 149.41 & 148.59 & 149.42 & 148.58 & 149.40 & 148.60 \\
12 & 149.41 & 148.59 & 149.42 & 148.58 & 149.40 & 148.60 \\
13 & 149.41 & 148.59 & 149.42 & 148.58 & 149.40 & 148.60 \\
14 & 149.41 & 148.59 & 149.42 & 148.58 & 149.40 & 148.60 \\
15 & 149.41 & 148.59 & 149.42 & 148.58 & 149.40 & 148.60 \\
16 & 149.41 & 148.59 & 149.42 & 148.58 & 149.40 & 148.60 \\
17 & 149.41 & 148.59 & 149.42 & 148.58 & 149.40 & 148.60 \\
18 & 149.41 & 148.59 & 149.42 & 148.58 & 149.40 & 148.60 \\
19 & 149.41 & 148.59 & 149.42 & 148.58 & 149.40 & 148.60 \\
20 & 149.41 & 148.59 & 149.42 & 148.58 & 149.40 & 148.60 \\
21 & 149.41 & 148.59 & 149.42 & 148.58 & 149.40 & 148.60 \\
22 & 149.41 & 148.59 & 149.42 & 148.58 & 149.40 & 148.60 \\
23 & 149.41 & 148.59 & 149.42 & 148.58 & 149.40 & 148.60 \\
24 & 149.41 & 148.59 & 149.42 & 148.58 & 149.40 & 148.60 \\
25 & 149.41 & 148.59 & 149.42 & 148.58 & 149.40 & 148.60 \\
\hline & & & & & &
\end{tabular}




\section{CONCLUSIONS}

This study found that by the application of (EWMA) classical control chart, exit four points out of control for the upper and lower limits control. The first point (149.43) was at the eight subgroup and the second point (149.68) was at the ten subgroup; these two points are out of the upper control limit. The third point (148.34) was at the fourteen subgroup and the fourth point (148.48) was at the twenty-four subgroup; these two points are out of the lower control limit. The presence of outliers in data of subgroups reduces the control chart's sensitivity because the control limits are stretched so that the outliers' detection becomes more difficult.

This study also found that through the application of (EWMA-SM), four points are out of the upper and lower limits of control. The first point (149.43) was at the eighth subgroup and the second two-point (149.68) was at the ten subgroups; these two points are out of the upper control limit. The third point (148.34) was at fourteen subgroups, and the fourth point (148.48) was at twenty-four subgroups; these two points are out of the lower control limit.

The control limits have been extended from the top and bottom sides of the (EWMA-SM) chart, making it less sensitive to the diagnosis of the shift in the mean process. The upper limit of control will be (149.42). The lower limit of control will be (148.58); its expansion could not diagnose other points out of control because of its expansion. However, it identified the same points mentioned in paragraph 3 ; the traditional control chart identified the same points.

The (EWMA-SMQ) chart detected extra points out of control from the bottom side, which is $(148,59),(148.59)$, (148.59) belonging to subgroups (15), (19), (21), respectively. In addition to the previous points identified by the two charts ((EWMA) \& (EWMA-SM) at subgroups (8), (10), (14), (24), respectively. Due to narrowness limits control of the (EWMA-SMQ) chart, three additional out of control points were detected, which were belonging to subgroups (15), (19), (21), respectively, which were mentioned in figure 3. Both the (EWMA-SM) \& (EWMA-SMQ) charts were able to diagnose the points out of control from the top, but only (EWMASMQ) chart was able to diagnose the shift in the process by detecting points from the bottom out of control, while (EWMA-SM) failed to diagnose them.

Widening the (EWMA-SM) chart limits so that the chart is less sensitive in detecting shifts in the mean. On the contrary, the (EWMA-SMQ) chart does not face this problem since its limits are computed based on the average sample interquartile range. Tight the limits of (EWMA-SMQ) so that the chart is more sensitive when outliers are present in data. (EWMASMQ) the chart is more robust than the (EWMA-SM) chart because outliers less influence the (EWMA-SMQ) chart compared to the (EWMA-SM) chart whose limits are computed based on the sample ranges. The (EWMA-SMQ) chart is superior to the (EWMA-SM) chart when only outliers present.

\section{REFERENCES}

[1] Z. Abbas, H. Z. Nazir, N. Akhtar, M. Riaz, and M. Abid, "On developing an exponentially weighted moving average chart under progressive setup: An efficient approach to manufacturing processes," Qual. Reliab. Eng. Int., vol. 36, no. 7, pp. 2569-2591, Nov. 2020, doi: 10.1002/qre.2716

[2] B. Zaman, M. Riaz, N. Abbas, and R. J. M. M. Does, "Mixed Cumulative Sum-Exponentially Weighted Moving Average Control Charts: An Efficient Way of Monitoring Process Location," Qual. Reliab. Eng. Int., vol. 31, no. 8, pp. 1407-1421, Dec. 2015, doi: 10.1002/qre. 1678

[3] M. R. Abujiya, M. H. Lee, and M. Riaz, "Improving the Performance of Exponentially Weighted Moving Average Control Charts," Qual. Reliab. Eng. Int., vol. 30, no. 4, pp. 571-590, Jun. 2014, doi: 10.1002 /qre. 1509 .

[4] A. Haq, "An improved mean deviation exponentially weighted moving average control chart to monitor process dispersion under ranked set sampling," J. Stat. Comput. Simul., vol. 84, no. 9, pp. 2011-2024, Sep. 2014, doi: 10.1080/00949655.2013.780059.

[5] N. Abbas, "Homogeneously weighted moving average control chart with an application in substrate manufacturing process," Comput. Ind. Eng., vol. 120, pp. 460-470, Jun. 2018, doi: 10.1016/j.cie.2018.05.009.

[6] J. M. Lucas and M. S. Saccucci, "Exponentially Weighted Moving Average Control Schemes: Properties and Enhancements," Technometrics, vol. 32, no. 1, pp. 1-12, Feb. 1990, doi: 10.1080/00401706.1990.10484583.

[7] M. A. Raza, T. Nawaz, M. Aslam, S. H. Bhatti, and R. A. K. Sherwani, "A new nonparametric double exponentially weighted moving average control chart," Qual. Reliab. Eng. Int., vol. 36, no. 1, pp. 68-87, Feb. 2020, doi: 10.1002/qre.2560.

[8] H. K. Sharaf, M. R. Ishak, S. M. Sapuan, N. Yidris, and A. Fattahi, "Experimental and numerical investigation of the mechanical behavior of full-scale wooden cross arm in the transmission towers in terms of load-deflection test," J. Mater. Res. Technol., vol. 9, no. 4, pp. 79377946, Jul. 2020, doi: 10.1016/j.jmrt.2020.04.069.

[9] S. H. Steiner, K. Grant, M. Coory, and H. A. Kelly, "Detecting the start of an influenza outbreak using exponentially weighted moving average charts," BMC Med. Inform. Decis. Mak., vol. 10, no. 1, p. 37, Dec. 2010, doi: 10.1186/1472-6947-10-37.

[10] H. K. Sharaf, M. R. Ishak, S. M. Sapuan, and N. Yidris, "Conceptual design of the cross-arm for the application in the transmission towers by using TRIZ-morphological chart-ANP methods," J. Mater. Res. Technol., vol. 9, no. 4, pp. 9182-9188, Jul. 2020, doi: 10.1016/j.jmrt.2020.05.129.

[11] H. K. Sharaf, S. Salman, M. H. Dindarloo, V. I. Kondrashchenko, A. A. Davidyants, and S. V. Kuznetsov, "The effects of the viscosity and density on the natural frequency of the cylindrical nanoshells conveying viscous fluid," Eur. Phys. J. Plus, vol. 136, no. 1, p. 40, Jan. 2021, doi: 10.1140/epjp/s13360-020-01026-y.

[12] N. A. Ajadi, O. Asiribo, and G. Dawodu, "Progressive mean exponentially weighted moving average control chart for monitoring the process location," Int. J. Qual. Reliab. Manag., vol. ahead-of-p, no. ahead-of-print, Dec. 2020, doi: 10.1108/IJQRM-05-2020-0138.

[13] M. Abid, A. Shabbir, H. Z. Nazir, R. A. K. Sherwani, and M. Riaz, "A double homogeneously weighted moving average control chart for monitoring of the process mean," Qual. Reliab. Eng. Int., vol. 36, no. 5, pp. 1513-1527, Jul. 2020, doi: 10.1002/qre.2641.

[14] H. Sharaf, N. A. Jalil, and S. Salman, "A simulation on the effect of ultrasonic vibration on ultrasonic assisted soldering of Cu / SAC 305 / Cu joint," J. Adv. Res. Appl. Mech., vol. 36, no. 1, 2017, [Online]. Available: http://www.akademiabaru.com/doc/ARAMV36_N1_P1_9.pdf.

[15] H. Z. Nazir, N. Abbas, M. Riaz, and R. J. M. M. Does, “A $\bar{A}$ comparative study of memory-type control charts under normal and contaminated normal environments," Qual. Reliab. Eng. Int., vol. 32, no. 4, pp. 1347-1356, Jun. 2016, doi: 10.1002/qre.1835.

[16] S.-L. Lu, "Novel design of composite generally weighted moving average and cumulative sum charts," Qual. Reliab. Eng. Int., vol. 33, no. 8, pp. 2397-2408, Dec. 2017, doi: 10.1002/qre.2197. 